\title{
The canonical approach to Finite Density QCD
}

\author{
Slavo Kratochvila* \\ Institute for Theoretical Physics, ETH Zürich, CH-8093 Zürich, Switzerland \\ E-mail: skratoch@phys.ethz.ch

\section{Philippe de Forcrand} \\ Institute for Theoretical Physics, ETH Zürich, CH-8093 Zürich, Switzerland \\ Physics Department, TH Unit, CERN, CH-1211 Geneva 23, Switzerland \\ E-mail: forcrandephys.ethz.ch
}

\begin{abstract}
We present a canonical approach to study properties of QCD at finite baryon density $\rho$, and apply it to the determination of the phase diagram of four-flavour QCD. For a pion mass $m_{\pi} \sim 350$ $\mathrm{MeV}$, the first-order transition between the hadronic and the plasma phase gives rise to a coexistence region in the $T$ - $\rho$ plane, which we study in detail. We obtain accurate results for systems containing up to 30 baryons and quark chemical potentials $\mu$ up to $2 T$. Our $T-\mu$ phase diagram agrees with the literature when $\frac{\mu}{T} \lesssim 1$. At larger chemical potential, we observe a "bending down" of the phase boundary. We characterise the two phases with simple models: the hadron resonance gas in the hadronic phase, the free massless quark gas in the plasma phase.
\end{abstract}

XXIIIrd International Symposium on Lattice Field Theory

25-30 July 2005

Trinity College, Dublin, Ireland

*Speaker. 


\section{Introduction}

The last few years have seen remarkable progress in the numerical study of QCD at finite chemical potential $\mu$. Still, the various methods $[1,2,3,4]$ suffer from systematic uncertainties, which limit their range of reliability to about $\frac{\mu}{T} \lesssim 1$.0. For a recent review, see Ref. [5]. We try to address this apparent limitation by using a canonical approach [6,7], where we focus on the matter density $\rho$, rather than the chemical potential. The method is particularly appropriate to explore fewnucleon systems at low temperature, and in principle, allows to study the bulk properties of nuclear matter and the nuclear interactions. Here, we extend its use and determine the phase boundary between the confined phase and the quark gluon plasma, as illustrated in Fig.1.
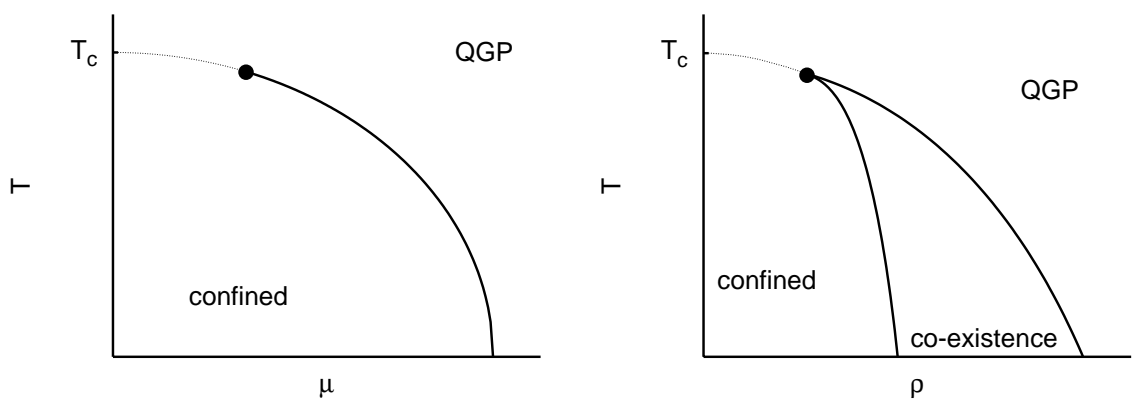

Figure 1: Sketch of the conjectured QCD phase diagram in the grand-canonical and canonical formalism.

The left figure shows a sketch of the conjectured phase diagram of QCD in the $T$ - $\mu$ plane. At small chemical potential, the phase transition is a rapid crossover, which ends in a second order endpoint, followed by a first order transition. Correspondingly, the right figure illustrates the transition in the $T-\rho$ plane. The first order transition is manifested in a co-existence region. In this proceedings, we describe how we identify the co-existence region and how we determine the phase diagram in the $T-\mu$ as well as in the $T-\rho$ plane.

\section{Partition Functions}

We construct the canonical partition function $Z_{C}(T, Q)$ by fixing the number of quarks $\hat{N}=$ $\int d^{3} \vec{x} \bar{\psi}(\vec{x}) \gamma_{0} \psi(\vec{x})$ to $Q$. We insert a $\delta$-function in the grand canonical partition function $Z_{G C}(T, \mu)$

$$
Z_{C}(T, Q)=\int[D U][D \bar{\Psi}][D \Psi] e^{-S_{g}[U ; T]-S_{F}[U, \bar{\Psi}, \Psi ; T, \mu]} \delta(\hat{N}-Q) .
$$

The $\delta$-function admits a Fourier representation $\delta(\hat{N}-Q)=\int d \bar{\mu}_{I} e^{i \bar{\mu}_{I}(N-Q)}$. We recognise $i \mu_{I}=$ $i \bar{\mu}_{I} T$ as an imaginary chemical potential and exploit the $\frac{2 \pi T}{3}$-periodicity [8] in $\mu_{I}$ of $Z_{G C}\left(T, \mu=i \mu_{I}\right)$

$$
Z_{C}(T, Q)=\frac{3}{2 \pi} \int_{-\frac{\pi}{3}}^{\frac{\pi}{3}} d \bar{\mu}_{I} e^{-i Q \bar{\mu}_{I}} Z_{G C}\left(T, i \bar{\mu}_{I} T\right) \underset{Q=3 B}{=} \frac{1}{2 \pi} \int_{-\pi}^{\pi} d\left(\frac{\mu_{I}}{T}\right) e^{-i 3 B \frac{\mu_{I}}{T}} Z_{G C}\left(T, i \mu_{I}\right) .
$$

Thus, the canonical partition functions are the coefficients of the Fourier expansion in imaginary $\mu$ of the grand canonical partition function. As a consequence of the $\frac{2 \pi T}{3}$-periodicity, the canonical partition functions are zero for non-integer baryon number $B=Q / 3$.

From the canonical partition functions, the grand canonical partition function can be reconstructed using the fugacity expansion (in fact a Laplace transformation)

$$
Z_{G C}(T, \mu) \underset{V \rightarrow \infty}{=} \int_{-\infty}^{\infty} d \rho e^{3 V \rho \frac{\mu}{T}} Z_{C}(T, \rho)=\int_{-\infty}^{\infty} d \rho e^{-\frac{V}{T}(f(T, \rho)-3 \mu \rho)}
$$


with the baryon density $\rho=\frac{B}{V}$ and the Helmholtz free energy density $f(T, \rho)=-\frac{T}{V} \log Z_{C}(T, \rho)$. The relation between baryon density and chemical potential can be expressed as $\langle\rho\rangle(\mu)$ or $\mu(\rho)$ :

$$
\langle\rho\rangle(\mu)=\frac{1}{Z_{G C}(T, \mu)} \int d \rho \rho e^{3 V \rho \frac{\mu}{T}} Z_{C}(T, \rho) \quad \text { or } \quad \mu(\rho)=\frac{1}{3} \frac{\partial f(\rho)}{\partial \rho} .
$$

While the first expression is exact in any volume, the second is obtained via a saddle point approximation (exact in the thermodynamic limit) and may have more than one solution when solving for the baryon density at a given chemical potential, see Fig.3. We discuss this issue in detail below.

\section{Method}

Following [9], we express the canonical partition function in a ratio, which can be measured by Monte Carlo simulation as an expectation value:

$$
\begin{gathered}
\frac{Z_{C}(B, \beta)}{Z_{G C}\left(\beta_{0}=\beta, \mu=i \mu_{I_{0}}\right)}=\frac{1}{Z_{G C}\left(\beta_{0}, i \mu_{I_{0}}\right)} \int[D U] e^{-S_{g}\left[U ; \beta_{0}\right]} \operatorname{det}\left(U ; i \mu_{I_{0}}\right) \frac{1}{2 \pi} \int_{-\pi}^{\pi} d\left(\frac{\mu_{I}}{T}\right) e^{-i 3 B \frac{\mu_{I}}{T}} \frac{\operatorname{det}\left(U ; i \mu_{I}\right)}{\operatorname{det}\left(U ; i \mu_{I_{0}}\right)} \\
=\left\langle\frac{1}{2 \pi} \int_{-\pi}^{\pi} d\left(\frac{\mu_{I}}{T}\right) e^{-i 3 B \frac{\mu_{I}}{T}} \frac{\operatorname{det}\left(U ; i \mu_{I}\right)}{\operatorname{det}\left(U ; i \mu_{I_{0}}\right)}\right\rangle_{\beta_{0}, i \mu_{I_{0}}} \equiv\left\langle\frac{\hat{Z}_{C}(U ; B)}{\operatorname{det}\left(U ; i \mu_{I_{0}}\right)}\right\rangle_{\beta_{0}, i \mu_{I_{0}}}
\end{gathered}
$$

where $Z_{G C}\left(\beta_{0}, i \mu_{I_{0}}\right)$ is the grand canonical partition function sampled by ordinary Monte Carlo methods, here for notational simplicity at $\beta_{0}=\beta$. The $\hat{Z}_{C}(U ; B)$ 's are the Fourier coefficients of the fermion determinant for a given configuration $\{U\}$. Although the average in Eq.(3.1) should be real positive, the individual measurements are complex, with a sometimes negative real part. This is how the sign problem manifests itself in our approach. Moreover, a reliable estimate depends on a good overlap of our Monte Carlo ensemble with the canonical sector $B$ at temperature $\beta$. We address this issue by following the idea of Ref. [1] and including both confined and deconfined configurations in our ensemble. Indeed, we supplement the ensemble at $\left(\beta_{c}(\mu=0), \mu=0\right)$ with additional critical ensembles at imaginary chemical potential, non-zero isospin chemical potential, and ensembles generated with an asymmetric Dirac coupling [4] - in principle, any ensemble is allowed. We then combine all this information about a particular canonical partition function $Z_{C}(B, \beta)$ by Ferrenberg-Swendsen reweighting [10].

The Fourier-coefficients of the determinant $\hat{Z}_{C}(U ; Q)$ are calculated exactly [9]. In the temporal gauge $\left(U_{4}(\mathbf{x}, t)=\mathbf{1}\right.$ except for $\left.t=N_{t}-1\right)$, the staggered fermion matrix $M$ in the presence of a chemical potential can be written in the form

$$
M=\left(\begin{array}{cccccc}
B_{0} & \mathbf{1} & 0 & \ldots & 0 & U_{N_{t}-1}^{\dagger} e^{-\mu a N_{t}} \\
-\mathbf{1} & B_{1} & \mathbf{1} & 0 & \ldots & 0 \\
0 & -\mathbf{1} & B_{2} & \mathbf{1} & 0 & \ldots \\
& & & \ldots & & \\
-U_{N_{t}-1} e^{\mu a N_{t}} & 0 & \ldots & 0 & -\mathbf{1} & B_{N_{t}-1}
\end{array}\right) \quad \longleftrightarrow \quad P=\left(\prod_{j=0}^{N_{t}-1}\left(\begin{array}{rr}
B_{j} & \mathbf{1} \\
\mathbf{1} & 0
\end{array}\right)\right) U_{N_{t}-1}
$$

where the $B_{i}$ 's contain all space-like contributions. Ref. [11] showed that the determinant can be computed for any chemical potential at the cost of diagonalising the so-called "reduced matrix" $P$. The determinant is given in terms of $P$ 's eigenvalues $\lambda_{1}, \ldots, \lambda_{6 V}$, where $V$ is the spatial volume:

$$
\operatorname{det} M(U ; \mu)=e^{3 V \mu a N_{t}} \prod_{i=1}^{6 V}\left(\lambda_{i}+e^{-\mu a N_{t}}\right)=\sum_{Q=-3 V}^{Q=3 V} \hat{Z}_{C}(U ; Q) e^{-Q \mu a N_{t}}
$$


Matching term by term, we then solve for the Fourier coefficients $\hat{Z}_{C}(U ; Q)$. This delicate step requires a special multi-precision library. The diagonalisation of the reduced matrix $P$ is computationally intensive and takes $\mathscr{O}\left(V^{3}\right)$ operations.

\section{Results}

We study the Helmholtz free energy $F(B) \equiv-T \log \frac{Z_{C}(B)}{Z_{C}(0)}$ in a theory of four degenerate flavours of staggered quarks with mass $m a=0.05\left(\frac{m}{T}=0.2, m_{\pi} \sim 350 \mathrm{MeV}\right)$ on a small $6^{3} \times 4$ lattice with volume $\sim(1.8 \mathrm{fm})^{3}$. For the quark mass we chose, the phase transition is first order at $\mu=0$, and presumably remains first order for all chemical potentials.

We "scan" the phase diagram by varying the baryon density at fixed temperature, see Fig.2. We measure $\frac{F(B)-F(B-1)}{3 T}$ and assume the validity of the saddle point approximation to equate this quantity with $\frac{\mu(B)}{T}$ following Eq. (2.4). This assumption will be tested in Fig.3.
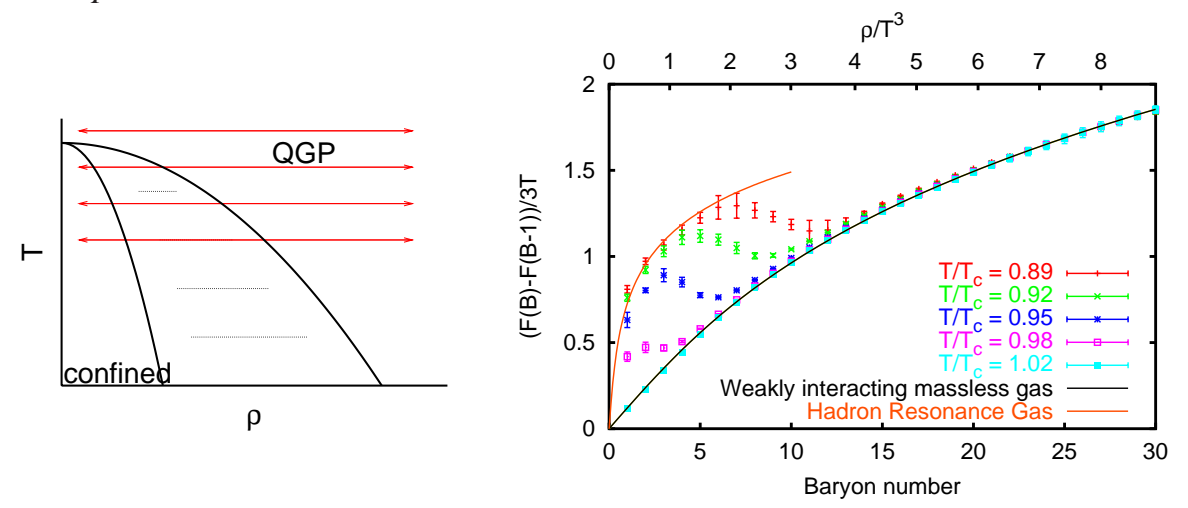

Figure 2: (left) A sketch of the "scans" in our phase diagram. (right) The derivative of the free energy at fixed temperature as a function of the baryon number (baryon density). In the saddle point approximation, the $y$-axis is $\frac{\mu}{T}=\frac{F(B)-F(B-1)}{3 T}$.

Note that accurate results are obtained, up to high densities $\left(>5\right.$ baryons $\left./ \mathrm{fm}^{3}\right)$ and large chemical potentials $\left(\frac{\mu}{T} \sim 2\right)$. The first order phase transition and the associated metastabilities are clearly visible in the "S-shape" of $\frac{\mu}{T}(B)$. The low-density regime can be reasonably well described by a simple hadron resonance gas Ansatz, $\frac{\rho}{T^{3}}=3 f(T) \sinh \left(3 \frac{\mu}{T}\right)$ with $f(T)$ as the only free parameter. The high-density regime almost corresponds to a gas of free massless quarks $\frac{\rho}{T^{3}}=$ $N_{f}\left(\frac{\mu}{T}\right)+\frac{N_{f}}{\pi^{2}}\left(\frac{\mu}{T}\right)^{3}$ when taking cut-off corrections [12] into account. The solid line in Fig.2(right) is obtained by fitting the linear and cubic terms in this expression. Instead of the free value 1 , the fitted coefficients are 0.82(2) and 1.94(6) respectively. Thus, the equation of state for the quarks in the plasma phase differs little from the Stefan-Boltzmann law. This has been observed also in [13] and [14]. Note that we find the same $\frac{\rho}{T^{3}}\left(\frac{\mu}{T}\right)$ dependence in the plasma phase at all temperatures.

For a given temperature $T$, we identify the boundaries $\rho_{1}$ and $\rho_{2}$ of the co-existence region and the critical chemical potential $\mu$ as follows. Equality of the free energy densities in the two phases, $f\left(\rho_{1}\right)-3 \mu \rho_{1}=f\left(\rho_{2}\right)-3 \mu \rho_{2}$, implies

$$
\int_{\rho_{1}}^{\rho_{2}} d \rho\left(f^{\prime}(\rho)-3 \mu\right)=0
$$

Since $f^{\prime}(\rho)$ is the quantity measured in Fig.2, we determine $\rho_{1}, \rho_{2}$ and $\mu$ by a "Maxwell construction" illustrated in Fig.3 (left) for the temperature $\frac{T}{T_{c}}=0.92$. The value of $\frac{\mu}{T}$ defining the horizontal 
line is adjusted to make the areas of the two "bumps" in the S-shape equal. ${ }^{1}$ The two outermost crossing points define $\rho_{1}$ and $\rho_{2}$, the boundaries of the co-existence region. Here, $\frac{\mu}{T}=1.06(2)$ is the value of the critical chemical potential.
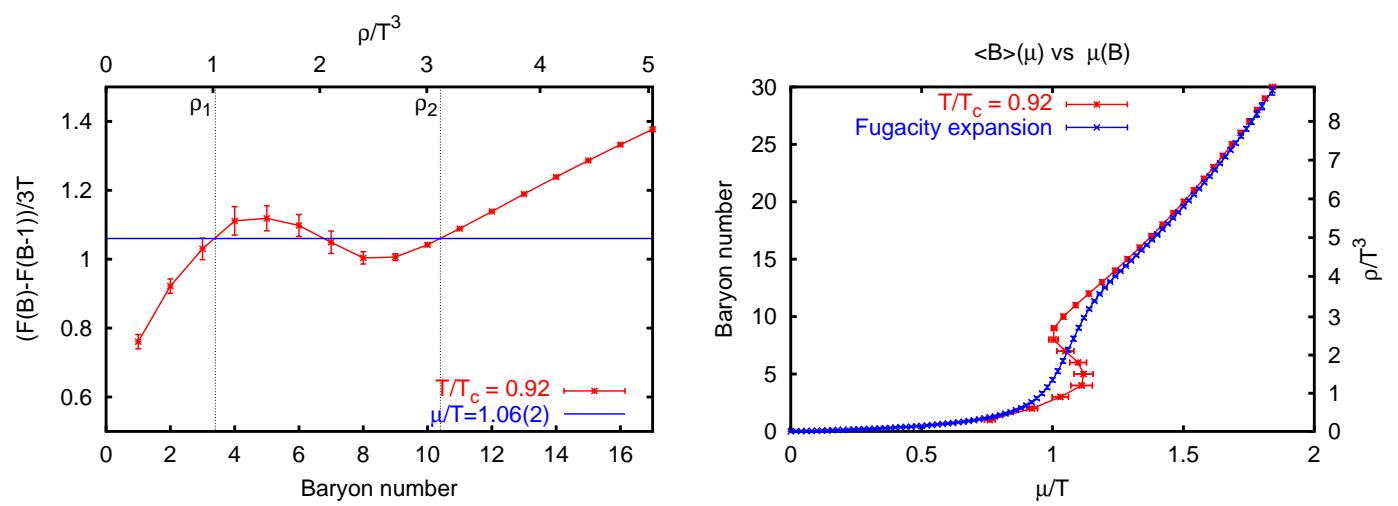

Figure 3: (left) The Maxwell construction allows to extract the critical chemical potential and the boundaries of the co-existence region. (right) Comparing the saddle point approximation (red) with the fugacity expansion (blue). Strong finite-size effects in the latter obscure the first-order transition.

We can cross-check this result by making use of the fugacity expansion Eq.(2.4), see Fig.3 (right). For a given chemical potential, we measure the baryon number $\langle B\rangle(\mu)$. We see a jump at the same value $\frac{\mu}{T} \approx 1.06$, but the rounding due to finite size effects is very strong. In contrast, our criterion for criticality (equality of the free energies) has exponentially small volume corrections.

In Fig.4 we present the phase diagrams in the $T-\mu$ as well as in the $T-\rho$ plane. On the left, we summarise results from various methods, all for the same theory: 4 flavours of staggered quarks with $a m=0.05, N_{t}=4$ time-slices; only the spatial volume varies as indicated. We have repeated (blue) the study of [1] (green), using multi-parameter reweighting on one ensemble generated at $\left(\beta_{c}, \mu=0\right)$. We identify the phase transition via the peak of the specific heat instead of LeeYang zeroes, and obtain consistent results. However, the "sign problem" dramatically grows with increasing chemical potential, as shown by the average sign in the figure. Moreover, our statistical error, based on jackknife bins as in [1], does not reflect the true inaccuracy.

The parabolic fit [3] is consistent with the black points [4]. Both methods perform an analytic continuation from imaginary $\mu$, for which the systematic errors are hard to quantify. Our new results are shown in red. There is no strong inconsistency with other results, but we observe a clear sign of bending down starting at $\frac{\mu}{T} \sim 1.3$. In fact this must happen, if the critical line is to reach the value $a \mu_{c}=0.35$ at $\beta=0$, predicted from a strong coupling analysis [15]. In the $T$ - $\rho$ plane, the densities at the boundaries of the co-existence region seem to remain constant for $T \lesssim 0.85 T_{c}$ already, with $\rho_{\mathrm{QGP}}=1.8(3) B / \mathrm{fm}^{3}$ and $\rho_{\text {confined }}=0.50(5) B / \mathrm{fm}^{3}$. The latter is a plausible value for the nuclear density in our 4-flavour, $m_{\pi}=350 \mathrm{MeV}$ QCD theory.

\section{Conclusions}

We study QCD in a canonical framework, which is promising for the study of few-nucleon systems at low temperature, but proves also capable of exploring high density regimes $(\mu / T \lesssim 2)$

\footnotetext{
${ }^{1}$ The area of each bump gives the free energy required to build two planar interfaces. The corresponding interface tension is $\sqrt{\sigma} \sim 35-45 \mathrm{MeV}$.
} 


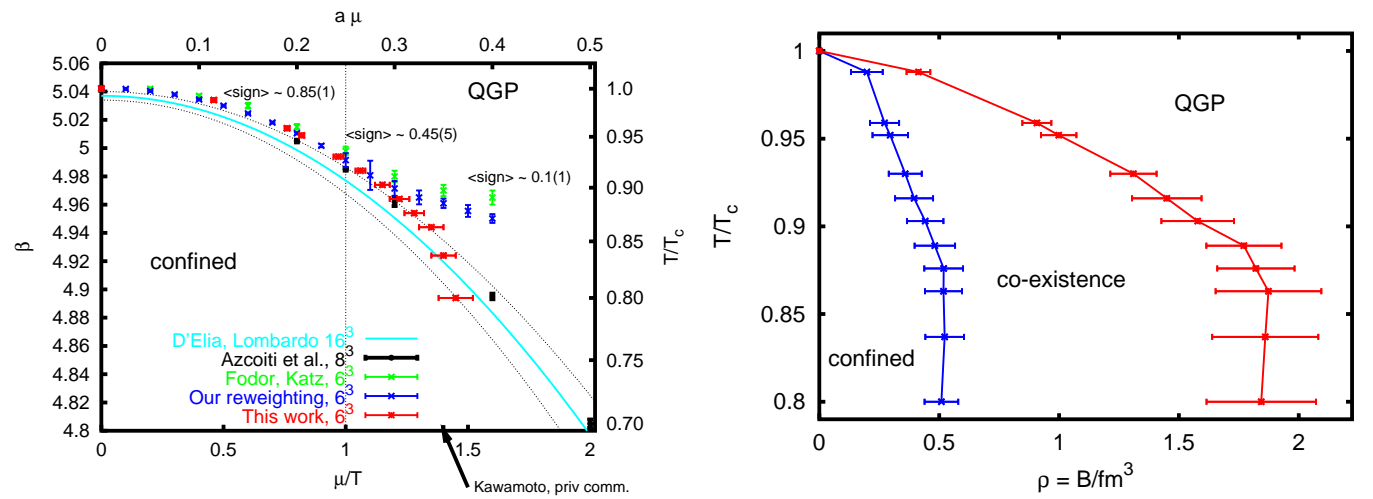

Figure 4: (left) The phase diagram in the $T$ - $\mu$-plane. (right) The phase diagram in the $T$ - $\rho$-plane.

at temperatures $T \gtrsim 0.8 T_{c}$. We have determined the phase boundary between the confined phase and the quark gluon plasma in both the $T$ - $\rho$ and the $T-\mu$ plane. In the latter, our results are in agreement with the literature, however we observe a bending down of the critical line at $\frac{\mu}{T} \sim 1.3$. The two phases can be rather well described by the hadron resonance gas at low densities and by a weakly interacting massless gas at high densities.

We thank the Minnesota Supercomputer Institute for computing resources.

\section{References}

[1] Z. Fodor and S. D. Katz, Phys. Lett. B 534, 87 (2002).

[2] C. R. Allton et al., Phys. Rev. D 66 (2002) 074507; R. V. Gavai and S. Gupta, Phys. Rev. D 68 (2003) 034506.

[3] M. D’Elia and M. P. Lombardo, Phys. Rev. D 67 (2003) 014505; P. de Forcrand and O. Philipsen, Nucl. Phys. B 642 (2002) 290; Nucl. Phys. B 673 (2003) 170.

[4] V. Azcoiti et al., hep-lat/0503010.

[5] O. Philipsen these proceedings.

[6] S. Kratochvila and P. de Forcrand, Prog. Theor. Phys. Suppl. 153 (2004) 330; S. Kratochvila and P. de Forcrand, hep-lat/0409072.

[7] A. Alexandru, M. Faber, I. Horvath and K. F. Liu, hep-lat/0507020.

[8] A. Roberge and N. Weiss, Nucl. Phys. B 275 (1986) 734.

[9] A. Hasenfratz and D. Toussaint, Nucl. Phys. B 371 (1992) 539.

[10] A. M. Ferrenberg and R. H. Swendsen, Phys. Rev. Lett. 61 (1988) 2635.

[11] P. E. Gibbs, Phys. Lett. B 172 (1986) 53.

[12] C. R. Allton et al., Phys. Rev. D 68 (2003) 014507.

[13] F. Csikor, G. I. Egri, Z. Fodor, S. D. Katz, K. K. Szabo and A. I. Toth, JHEP 0405 (2004) 046.

[14] C. R. Allton et al., Phys. Rev. D 71, 054508 (2005).

[15] N. Kawamoto, private communication and in writing. 